\title{
Vocal Change Patterns During a Teaching Day: Inter- and Intra-subject Variability
}

\author{
*†Angélique Remacle, *־Maëva Garnier, *'†Silvain Gerber, ¥Claire David, and ¥Caroline Petillon, *Saint Martin d'Hères \\ Cedex, †Grenoble, and $\ddagger$ Lyon, France
}

\begin{abstract}
Summary: Objectives. To describe the mean voice changes of 22 female teachers during a typical workday, examine the inter- and intra-subject variability, and establish a typology of different voice patterns during the workday.

Methods. For each participant, fundamental frequency (F0), harmonics-to-noise ratio (HNR), jitter, and shimmer were measured on sustained vowels at the beginning and at the end of the workday, at three different times during the school year.
\end{abstract}

Results. The group mean pattern showed significant increases in F0 and HNR during the workday and significant decreases in jitter and shimmer. However, considerable inter- and intra-subject variability was observed. Based on the variation in the acoustic parameters during the workday, three different voice patterns were identified. The first is characterized by a greater F0 increase during the day, interpreted as a common, appropriate adaptation to vocal load. The second is characterized by a greater increase in HNR during the day and greater decreases in jitter and shimmer, interpreted as hyperfunctional voice production. The third is characterized by greater decreases in F0 and HNR and greater increases in jitter and shimmer, suggesting acute inflammation or muscle fatigue following the workday.

Conclusions. The observed variety of vocal patterns during the workday emphasizes the need to study this phenomenon individually and target different types of behaviors to develop tailored prevention and treatment methods.

Key Words: Vocal fatigue-Vocal loading-Vocal hyperfunction-Acoustic analysis-Teachers.

\section{INTRODUCTION}

During a teaching day, a teacher's voice is frequently challenged. One major stressor that compromises optimal laryngeal function is vocal loading, ${ }^{1}$ which is closely related to the amount of voicing. ${ }^{2}$ The quantification of teachers' daily voice use has revealed particularly long phonation duration, high voice frequency, and sound pressure level. ${ }^{3,4}$ Such intensive use leads to objective voice changes and subjective symptoms, such as vocal fatigue, increased perceived phonatory effort, and a feeling of having a tired throat. ${ }^{1,5,6}$ The time when these symptoms appear or disappear is difficult to estimate because vocal recovery differs across speakers ${ }^{7.8}$ These symptoms can become chronic and lead to dysphonia or laryngeal changes if the speaker takes insufficient vocal rest or engages in maladaptive compensatory strategies. $^{7}$

The biomechanical and physiological mechanisms induced by vocal load are not yet fully understood. Titze suggested several hypotheses. ${ }^{910}$ First, some symptoms may be related to the mechanical stress placed on the vocal folds (tension, repeated collisions, and frictions). This stress can cause phonotrauma such as edema or nodules. It can also cause tissue inflammation, reduced blood flow, and a rise in vocal fold temperature. ${ }^{9}$ The subsequent increased vocal fold stiffness and viscosity may

\footnotetext{
Accepted for publication March 13, 2017.

Present address: Unité Logopédie de la Voix, Faculté de Psychologie, Logopédie et sciences de l'Éducation, Université de Liège, Rue de l'Aunaie, 30 (B38), 4000 - Sart Tilman, Belgium

From the *CNRS, GIPSA-lab, 11 rue des Mathématiques, Saint Martin d'Hères Cedex, France; †University Grenoble-Alpes, Grenoble, France; and the $¥$ Institut des Sciences et Techniques de Réadaptation, Université Claude Bernard Lyon 1, Lyon, France.

Address correspondence and reprint requests to Angélique Remacle, CNRS, GIPSA-lab,

11 rue des Mathématiques, Grenoble Campus BP46, F-38402 Saint Martin d'Hères Cedex,

France. E-mail: Angelique.Remacle@ulg.ac.be

Journal of Voice, Vol. $\square \square$, No. $\square \square$, pp. $\square-\square \square$

0892-1997

(C) 2017 The Voice Foundation. Published by Elsevier Inc. All rights reserved.

https://doi.org/10.1016/j.jvoice.2017.03.008
}

explain the raised phonation threshold pressure (ie, the minimal subglottal pressure to initiate and maintain vibration) and the perceived increase in phonatory effort observed in some speakers. ${ }^{11,12}$ Vocal symptoms may also result from neuromuscular fatigue of the intrinsic or extrinsic laryngeal muscles, making it difficult to maintain vocal fold tension and stability of the laryngeal posture. ${ }^{913}$ Another mechanism following intensive voice use may be reduced blood circulation, which impairs the dissipation of heat away from the vocal folds, the removal of lactic acid from muscles, the replacement of oxygen, and the supply of energy resources. ${ }^{9,13}$

Intrinsic and extrinsic factors can influence vocal load by accelerating or delaying the appearance of its symptoms. Intrinsic factors relate to the subject's voice use (phonation type, voice quality, duration of phonation, fundamental frequency (F0), and intensity of voice), ${ }^{1}$ biological factors (gender, age, genetics, hormones, and vocal fold biochemistry), ${ }^{1}$ and aerobic conditioning. ${ }^{14}$ Extrinsic factors relate to the environment influencing the voice, for instance room acoustics, humidity, ${ }^{15}$ and background noise. ${ }^{4}$

Two main approaches have been used in previous studies to investigate the appearance of and recovery from changes because of vocal load in both healthy and dysphonic individuals. First, most such studies were conducted under laboratory conditions ${ }^{2,11,12,14,16-21}$ and used endurance tasks believed to challenge the vocal mechanism, such as prolonged speaking, speaking against background noise, sustaining vowels, and singing. ${ }^{1}$ These tasks are referred to as vocally fatiguing tasks, vocal loading tasks, or vocal-loading tests. Second, a few studies have explored vocal load under realistic field conditions. ${ }^{3,5,6,22,23}$ In these studies, speakers' responses to loading were examined in real-life situations, usually with individuals who had high vocal demands or were at risk of voice disorders. Given their extensive voice use, professional voice users, and especially teachers, have frequently been studied. The motivation for studying these professionals 
stems from growing evidence that their voice disorders represent a common, and costly, problem for the workforce, particularly in the case of teachers.

Common measurements for functional assessment of voice include auditory-perceptual assessment, laryngeal visualization, aerodynamics, acoustics, and subjective self-evaluation by the speaker. ${ }^{24}$ Specific acoustic descriptors, such as F0, harmonicsto-noise ratio (HNR), period perturbation (jitter), and amplitude perturbation (shimmer), have been shown to be relevant, reliable parameters for clinical voice assessment. ${ }^{24}$ These measurements have also been used to assess the changes following vocal load. As presented in a recent review, ${ }^{1}$ previous studies aiming to describe voice conditions following vocal demand, which used a variety of methods, populations studied, and measurements, yielded mixed and contradictory observations. ${ }^{13,14}$ For instance, some studies showed a significant increase in F0 after prolonged voice use, whereas others reported a significant decrease. ${ }^{1,2,5,6,17,19,25}$ A similar discrepancy between studies has been observed for other voice descriptors such as HNR,,$^{1,2,17,25}$ jitter, $^{1,2,6,17,19}$ and shimmer. ${ }^{1,2,6,17,25}$

An explanation of this discrepancy may be that most of these studies described the average response of a specific group of individuals to vocal demand without making inter- and intraindividual distinctions. However, the variability of clinical cases of subjects experiencing vocal challenges emphasizes the need to study this phenomenon at the individual level. Furthermore, clinical observations have shown that the same individual may produce different responses to vocal loading at different times, depending on several underlying factors such as the environment, general fatigue, psycho-emotional factors, and medical conditions influencing voice (eg, asthma, seasonal allergies, ear, nose, throat (ENT) infections, or laryngopharyngeal reflux). In their literature reviews, Welham and Maclagan ${ }^{13}$ and Solomon highlighted the need to study individual differences in responses to vocal fatigue in ecological conditions.

Accordingly, the present study investigated the individual voice patterns of 22 teachers during a workday, at three different times in the school year. To this end, four acoustic parameters that are easily applicable in a naturalistic situation were selected to provide objective, noninvasive measures of vocal function: F0, HNR, jitter, and shimmer. Based on the changes in these four acoustic parameters, our aims were (1) to describe the mean voice changes of a group of 22 teachers during a workday, (2) to examine the intra-speaker variability at three different times of the year and the inter-speaker variability across the 22 individuals, and (3) to establish a typology of voice patterns during a typical workday, corresponding to different changes in the four acoustic parameters during the day.

\section{MATERIALS AND METHODS}

\section{Participants}

Twenty-two female teachers participated in this study (mean age: 31, standard deviation [SD]: 6, range: 24-42); they are referred to as S1 to S22. All of them worked in the Rhône-Alpes region of France. Female kindergarten and elementary school teachers were recruited because they have very vocally demanding professions. ${ }^{3}$

Before the experiment, each participant signed a consent form, and the study was approved by the local education authority. The following inclusion criteria were established: participants must be women, with less than 5 years of teaching experience, no previous laryngeal surgery, no previous speech therapy for a voice problem, and no voice problem detected by the experimenter at the time of the study based on perceptual analysis. Perceptual analyses were performed by a speech therapist specializing in voice (A.R.), a researcher (M.G.), and two master's students in speech therapy (C.D. and C.P.), based on the recording of sustained vowels, reading of texts, and spontaneous speech.

Given the strict inclusion criteria and the absence of financial compensation for their participation, 22 teachers were recruited for the study. Seven participants were kindergarten teachers and 15 were elementary schoolteachers. Their class size ranged from 12 to 31 children (mean: 24). Their mean working time per week was 6.5 half-days of teaching (SD: 1.7). Background information collected with a questionnaire showed that 13 participants had experienced problems with their voice during their career and six of them had been on sick leave for voice problems. Five participants reported a modification of their voices during their career: lower-pitched voice (S4), increased roughness (S5 and S22), difficulty reaching high notes while singing (S10), and less vocal fatigue (S15). One participant was a smoker (S4) and another was an occasional smoker (S5). Two participants sang nonprofessionally during their leisure time (S4 and S8). However, all the participants presented a normal voice at the time of the study.

\section{Material, task, and data extracted}

For each participant, five repetitions of the sustained vowel [a], produced at a comfortable pitch and intensity, were recorded twice during the workday: in the morning before the first lesson and in the evening after the last lesson. This procedure was repeated at three different times during the school year: in October (T0), December (T1), and February (T2).

Voice productions were recorded using a portable digital stereo recorder (ZOOM H1, Tokyo, Japan) and a headset cardioid microphone (Shure WH20, Niles, IL) in each teacher's classroom but in quiet conditions (before the children arrived and after they left). The input level was set individually to avoid saturation. The audio signal was recorded in .wav format, with a sampling frequency of $44.1 \mathrm{kHz}$ and 16 bits.

Acoustic analysis was conducted using the freeware Praat (Boersma \& Weenink, Amsterdam, The Netherlands). ${ }^{26}$ For each sustained vowel, the mean F0 (Hz) was measured, using an autocorrelation method. The HNR (dB), local jitter (in \%), and local shimmer (in \%) were also measured.

Then, the mean values of these parameters over the five repetitions produced in the morning and the evening at each time of year (T0, T1, and T2) were considered. Because this paper aims to document voice patterns during a typical workday, the differences between these mean values from morning to evening (referred to as $\Delta \mathrm{F} 0, \Delta \mathrm{HNR}, \Delta \mathrm{jitter}, \Delta$ shimmer) were calculated for each participant, at each time of year. A positive $\Delta$ suggests 
an increase from the morning to the evening, whereas a negative $\Delta$ suggests a decrease.

\section{Statistical analyses}

Several statistical analyses were conducted using R software (R Foundation for Statistical Computing, Vienna, Austria). The significance level was set at 0.05 .

First, the significance of each acoustic parameter change during a workday ( $\Delta$ values) was tested for the whole group. An analysis of variance from a mixed model of the data was conducted using the $\mathrm{R}$ package lme, considering the five repetitions of the vowels, and taking into account not only fixed effects (workday and time of year factors) but also a random effect (participant factor). The simplest model was selected to best explain the variance in each acoustic parameter, using a descending approach based on the minimization of the Bayesian information criterion and on the likelihood ratio test (LRT). Hypotheses about the model's normality and homoscedasticity were validated by looking at the residuals graphs. After examining the effects of the interaction terms remaining in the simplified model, the mean contrast between the values measured in the morning and the evening was tested using the multcomp package in $\mathrm{R}$ and applying Bonferroni adjustments for multiple comparisons.

Second, the change in acoustic parameters during a workday was described for each speaker. The mean intra-speaker variability across the three times of year and the mean interspeaker variability between speakers were detailed.

Third, significantly different voice patterns were distinguished among all the teaching days. Using the hclust package in $\mathrm{R}$, an ascending hierarchical classification was run from a combination of the four acoustic parameters' changes ( $\Delta$ values) for each workday. Because of the substantial response variability observed within subjects, the 66 workdays were considered on the same level, as if independent from each other. The Euclidean distance and Ward's aggregation index were used to cluster the 66 workdays: a dendrogram represented the result of this classification. The number of groups in this classification was chosen, considering the decrease in homogeneity between two successive clusters resulting from the algorithm, and more concretely, looking at the plot of the gains of intra-category inertia. For this dataset, three categories appeared to be relevant to significantly distinguish among different types of patterns during a teaching day. These three types of patterns and their distinctive features were characterized: first, the significance of the changes in acoustic parameters during a workday was tested for each group; then, the mean for each group was compared with the mean for the 66 workdays using mean comparison tests.

\section{RESULTS}

\section{Changes in acoustic parameters during the workday} for the whole group

Overall, the results of the analysis of variance examining the changes from morning to evening indicated significant changes for each of the four acoustic parameters.

No significant interaction between the time of year and workday factors was observed for $\Delta \mathrm{HNR}$ (LRT: $\mathrm{df}=2$, LRatio $=3.7$,
$P=0.16)$, meaning that the significant increase in that parameter observed over the workday $(\triangle \mathrm{HNR}=1.9 \pm 0.3 \mathrm{~dB}, P<0.0001)$ was somewhat comparable at all three measurement times. On the other hand, a significant interaction between the time of year and workday factors was observed for $\Delta \mathrm{F} 0$ (LRT: $\mathrm{df}=2$, LRatio $=11.1, P=0.004), \Delta$ jitter $($ LRT: $\mathrm{df}=2$, LRatio $=6.9$, $P=0.032$ ), and $\Delta$ shimmer (LRT test: $\mathrm{df}=2$, LRatio $=8.5$, $P=0.01$ ), meaning that the changes in those parameters over the workday varied significantly with the time of year. However, the changes in these parameters over the whole group always followed the same direction at all three times: on average, F0 increased significantly during the workday $(\Delta \mathrm{F} 0=9 \pm 1 \mathrm{~Hz}$, $P<0.001)$, whereas jitter and shimmer decreased significantly $(\Delta$ jitter $=-0.19 \pm 0.03 \%, P<0.001 ; \Delta$ shimmer $=-0.74 \pm 0.17 \%$, $P<0.001)$.

\section{Changes in acoustic parameters during the workday for each speaker}

Figure 1 summarizes each participant's mean change during the workday ( $\Delta \mathrm{F} 0, \Delta \mathrm{HNR}, \Delta \mathrm{jitter}, \Delta$ shimmer), as well as the intraspeaker variability across the three times of year.

Considerable inter-speaker variability was observed in the changes in acoustic parameters during the workday $(\Delta \mathrm{F} 0$ $\mathrm{SD}=20.7 \mathrm{~Hz} ; \Delta \mathrm{HNR} \mathrm{SD}=4.1 \mathrm{~dB} ; \Delta$ jitter $\mathrm{SD}=0.41 \%$; $\Delta$ shimmer $\mathrm{SD}=2.6 \% \mathrm{a})$. This variability shows that the subjects do not all present the same vocal pattern during the workday. Figure 1 shows that F0 systematically increased during the workday at all three times for only eight speakers (left frame), whereas 13 speakers showed a varying trajectory for F0, and one speaker (S10) spoke systematically at a lower pitch at the end of the workday (right frame). HNR systematically increased during the workday for nine speakers (left frame), showed a varying trajectory for 11 speakers, and systematically decreased for two speakers (S10 and S18; right frame). Finally, both jitter and shimmer systematically decreased during the workday for nine speakers (left frame), showed a varying trajectory for 11 speakers, and systematically increased for two speakers (S10 and S18; right frame). This variability stems from the fact that some participants have a systematic but small change in these acoustic parameters during the day, whereas others demonstrate a varying but large change. Furthermore, these results show that the changes in these acoustic parameters are sensitive not only to inter-subject variability, but also to a nonnegligible intra-subject variability across the three times of year (mean intra-speaker variability in $\Delta \mathrm{F} 0: 15.2 \mathrm{~Hz} ; \Delta \mathrm{HNR}: 2.9 \mathrm{~dB}$; $\Delta$ jitter: $0.19 \%$; $\Delta$ shimmer: $1.4 \%$ ).

\section{Typology of voice changes during a workday}

The ascending hierarchical classification of the 66 workdays revealed three distinct groups, corresponding to three significantly different types of responses to a workday. Figure 2 presents the dendrogram of this classification and the distribution of the 66 workdays (22 participants $\times 3$ times of year) into these three groups.

\footnotetext{
aThese standard deviations were computed from each of the 22 participants' mean changes during the workday over the three times of year.
} 

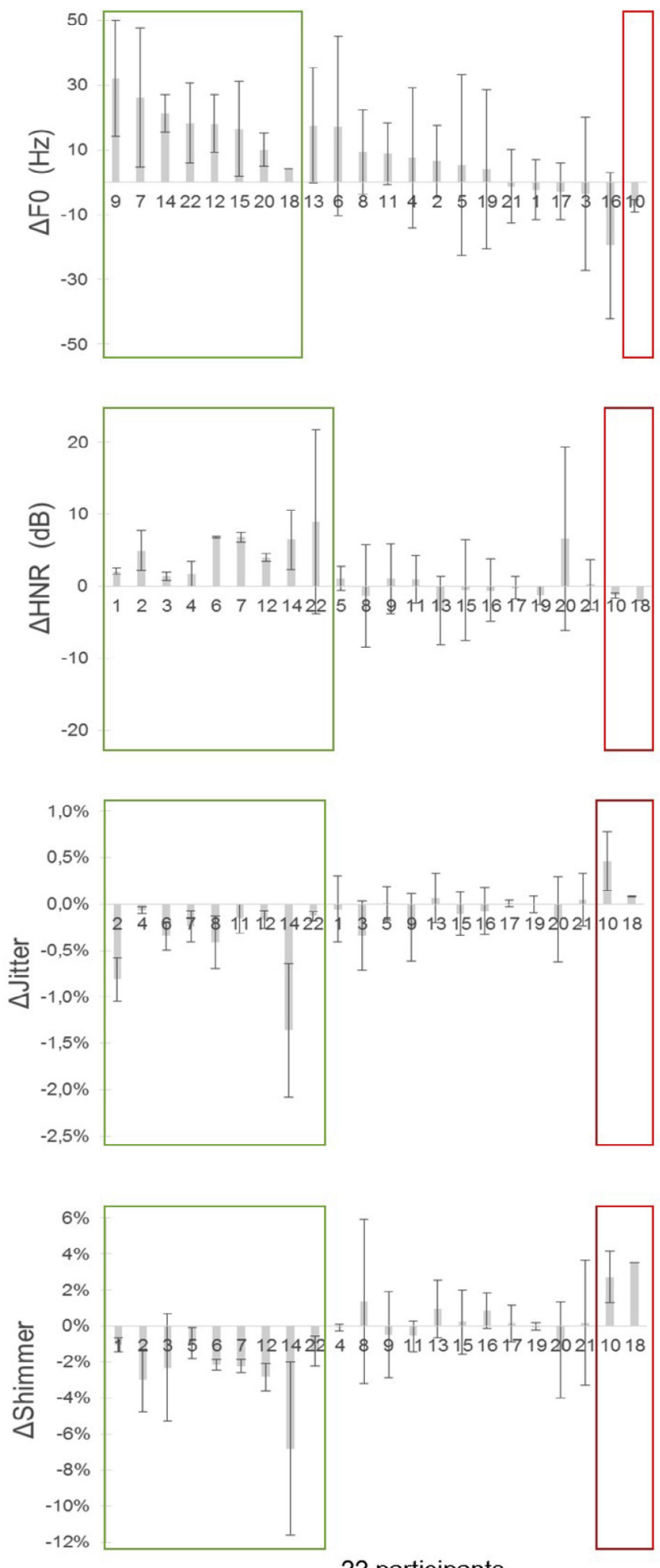

22 participants

FIGURE 1. Mean changes in the four acoustic parameters during the day $(\Delta)$ for each participant. Participants are represented by their number (1-22) on the x-axis. Error bars indicate standard deviation (ie, intraspeaker variation across the three times of year). Speakers who systematically behave in the same way as the group mean are presented in the left frame of each graph (in green); the central part of the graph represents speakers with varying patterns across the three times of year; the right frame of the graph (in red) includes the speakers who systematically behave in the same way but contrary to the group mean. (For interpretation of the references to color in this figure legend, the reader is referred to the Web version of this article.)
The first group included 37 workdays. This group pattern during the workday is characterized by an increase in F0 $(P<0.001)$ and HNR $(P<0.001)$, and a decrease in jitter $(P<0.001)$ and shimmer $(P<0.001)$ (see Figure 3$)$. The mean comparison test showed that group 1 distinguished itself from the mean for the 66 workdays only by a significantly greater $\Delta \mathrm{F} 0$ $(+4.7 \mathrm{~Hz}$ on average, $P<0.0001)$. The second group included 6 workdays. This group pattern during the workday is characterized by an increase in F0 $(P=0.0042)$ and HNR $(P<0.001)$, and a decrease in jitter $(P<0.001)$ and shimmer $(P<0.001)$ (see Figure 3). The mean comparison test showed that group 2 distinguished itself from the mean for the 66 workdays by a significantly greater $\Delta \mathrm{HNR}$ following the workday $(+4.6 \mathrm{~dB}$ on average, $P<0.0001)$, and a significantly lower $\Delta$ jitter $(-5.0 \%$ on average, $P<0.0001)$ and $\Delta$ shimmer $(-4.9 \%$ on average, $P<0.0001)$. The third group included 23 workdays. This group pattern is characterized by a decrease in $\mathrm{F} 0(P=0.002)$ and HNR $(P=0.0299)$, and an increase in jitter $(P=0.031)$ and shimmer $(P<0.001)$ (see Figure 3$)$. The mean comparison test showed that group 3 distinguished itself from the mean for the 66 workdays by a significantly lower $\Delta \mathrm{F} 0(-5.7 \mathrm{~Hz}$ on average, $P<0.0001)$ and $\Delta \mathrm{HNR}(-4.4 \mathrm{~dB}$ on average, $P<0.0001)$ following the workday, and by a significantly greater $\Delta$ jitter $(+4.6 \%$ on average, $P<0.0001)$ and $\Delta$ shimmer $(+5.0 \%$ on average, $P<0.0001)$.

\section{DISCUSSION}

\section{Mean voice changes during a workday}

Our first objective was to describe the mean voice changes of the 22 female teachers during a workday. In agreement with previous studies, the results showed a significant mean increase in F0, ${ }^{2,5,6,17,19}$ decrease in jitter, ${ }^{6,19}$ and decrease in shimmer ${ }^{2,6,17}$ after prolonged voice use. On the other hand, a significant mean increase in HNR was observed, although the literature has generally reported a variable or nonsignificant change in that parameter. ${ }^{1,2,17,25}$

\section{Inter- and intra-speaker variability}

The second objective of the study was to describe the inter- and intra-individual variability in voice patterns during a workday. For the four acoustic parameters, large standard deviations were observed within and across individuals. Consequently, and despite their statistical significance, the mean changes reported above are far from being generalizable across all speakers at all three times of year. Figure 1 clearly illustrates how some speakers systematically showed an increase in a given acoustic parameter during the workday at all three times of year, whereas others systematically showed a decrease, and the majority of them showed varying patterns according to the teaching day.

Several hypotheses can be formulated to explain this variability. A teacher's vocal demands may differ across the three times of year, depending on school activities, children's behavior, and environmental factors. An individual's sensitivity to vocal load may vary from one day to another, depending on environmental, physiological, and psycho-emotional factors (ie, intrasubject variability). Some people may be more sensitive to vocal load than others, depending on their anatomy, genetics, state of 


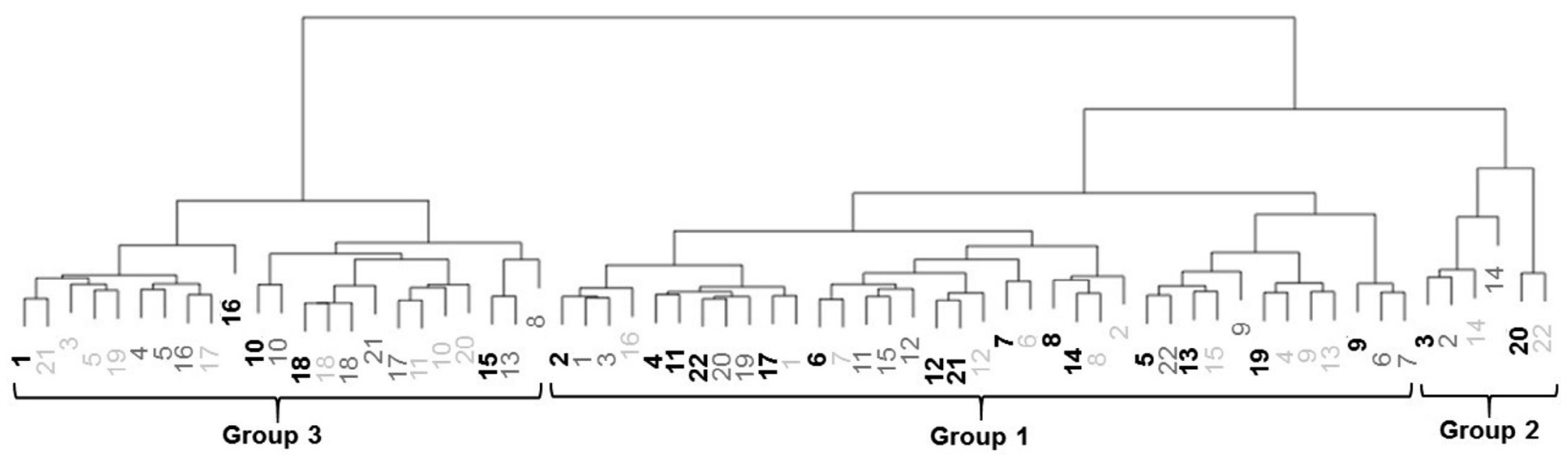

FIGURE 2. Dendrogram representing the distances between the 66 workdays, calculated from the combination of $\Delta \mathrm{F} 0, \Delta \mathrm{HNR}, \Delta \mathrm{jitter}$, and $\Delta \mathrm{shimmer}$ during these workdays. Three types of voice change pattern during the workday were distinguished (groups 1, 2, and 3). Participants are represented by their number (1-22); the time of the year is indicated by the color code (T0 in black, T1 in dark gray, and T2 in light gray).

health, vocal training, ${ }^{12}$ technique and experience (ie, intersubject variability). People may also demonstrate varying degrees of awareness and attention to vocal symptoms, and therefore react differently to them. Regardless of the causes of this great interand intra-speaker variability, this observation highlights the necessity of going beyond the interpretation of the whole group's pattern and encourages us to identify different types of change patterns during a workday.

\section{Typology of voice changes during a workday}

The third objective of this study was to distinguish among different types of voice changes during a typical workday. Based on the changes in acoustic parameters from morning to evening across 66 workdays, three significantly different types of responses emerged. Taking into account the intra-subject variability described above, a given individual may belong to different groups at different times of year.

The first type of voice change pattern (group 1) characterized most of the workdays (56\%). This group distinguished itself from the mean change for the 66 workdays only by a significantly greater F0 increase during the day. Physiologically, F0 is regulated by the combined action of laryngeal muscles and lung pressure. The adjustment of laryngeal muscles influences the vocal folds' length, stiffness (muscle or cover stiffness), vibrating mass, and therefore vibration frequency. ${ }^{10}$ Subglottal pressure also influences the vibration amplitude of the vocal folds and its associated tension, ${ }^{27}$ affecting the vibration frequency. Based on these physiological principles, we hypothesize that an F0 rise during the workday may be the consequence of increased tension in the larynx and paralaryngeal areas. Like previous studies, ${ }^{2,5,6,28}$ we consider that a reasonable F0 increase may be a common and appropriate physiological adaptation of the phonatory function to vocal loading. Consequently, the behavior identified in group 1 may be interpreted as an appropriate response to a teaching day.

A second type of voice pattern (group 2) was observed for $9 \%$ of the workdays. This second group distinguished itself from the mean change by a significantly greater increase in HNR following the workday and a significantly greater decrease in jitter and shimmer. Several hypotheses can be proposed to explain these variations. An increase in HNR following the workday reflects more harmonicity in the voice (ie, less noise or inharmonic content in the voice spectrum ${ }^{26}$ ), which suggests increased glottal adduction potentially accompanied by increased subglottal pressure. Previously, perceptual evaluations showed a significant decrease in voice breathiness following vocal loading, suggesting an increase in voice harmonic richness, resulting from decreased laryngeal air leakage or increased laryngeal tonus. ${ }^{20}$ The decrease in jitter can be simply explained by the increased F0. Indeed, the calculation method for jitter implies a direct negative correlation between these two parameters. ${ }^{29}$ However, a combined decrease in jitter and shimmer may also result from reduced variations or asymmetries in muscle tension or reduced fluctuations in subglottal pressure or glottal airflow. ${ }^{30}$

As in a previous study of teachers' voice changes following a working day, ${ }^{6}$ a combined increase in F0 and HNR and a decrease in perturbation values suggest increased laryngeal tension and more hyperfunctional voice production following vocal load. Roy et al defined vocal hyperfunction as "excessive, imbalanced, or dysregulated laryngeal muscle activity, force, or tension accompanying voice production." ${ }^{31}$ Excessive laryngeal tension or activation of muscles suggests an inappropriate response to vocal challenge, at a level that exceeds task demands, or the "engagement" of compensatory strategies. ${ }^{7}$ This hyperfunctional response may result from an inefficient phonatory technique, vocal misuse, vocal abuse, or insufficient periods of vocal rest, potentially leading to chronic vocal fatigue. Vocal hyperfunction is known to cause a variety of disorders, such as vocal nodules, polyps, or primary muscle tension dysphonia. ${ }^{31}$

A third type of voice pattern (group 3) characterized $35 \%$ of the workdays. This third group distinguished itself from the mean change by a significant decrease in F0 and HNR following the workday, along with a significant increase in jitter and shimmer. One possible explanation for these changes in acoustic parameters may be the development of an acute inflammatory response following the workday. Previous videostroboscopic studies reported that some subjects develop vocal fold edema following prolonged voice use. ${ }^{25,32}$ The effect of inflammatory response, 

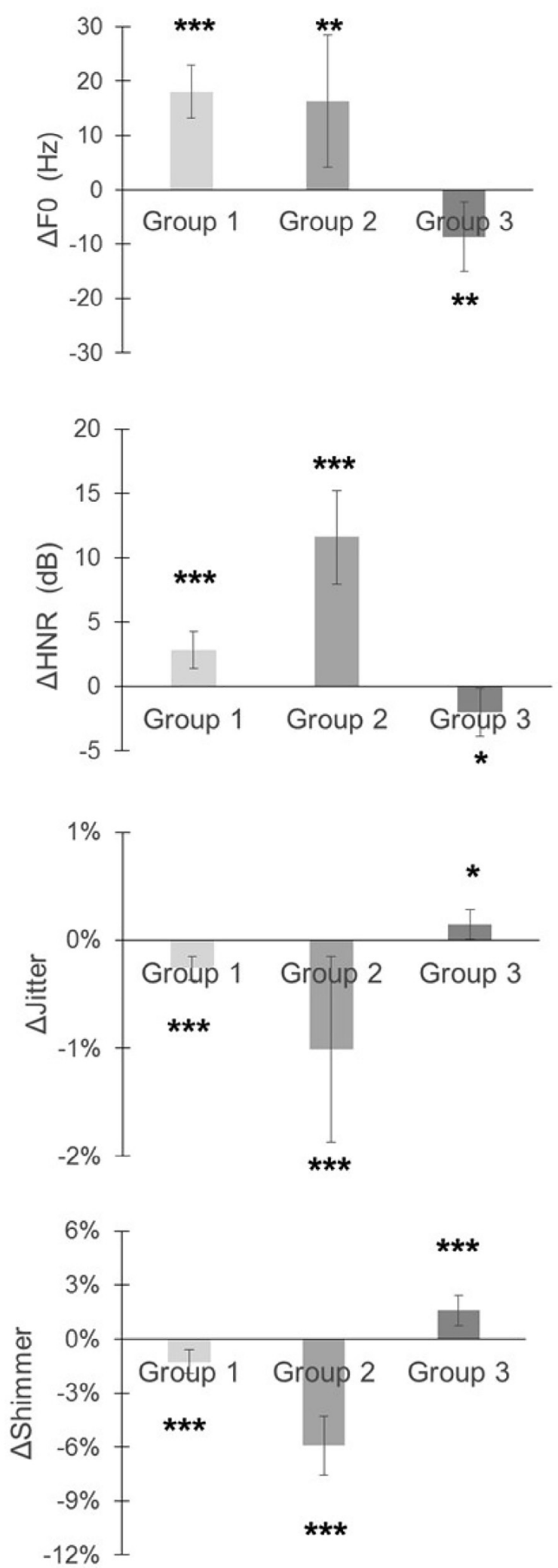

FIGURE 3. Mean changes in the acoustic parameters from morning to evening $(\Delta)$ for the 3 groups, resulting from the ascending hierarchical classification. Error bars represent the confidence intervals estimated from the statistical model. Asterisks represent the significance level of the workday effect $(* P \leq 0.05 ; * * P \leq 0.01 ; * * * P \leq 0.001)$. such as edema, is to add weight to the vocal folds, which decreases their frequency of vibration, ${ }^{33}$ resulting in decreased F0. Inflammation can also degrade the vocal folds' vibratory qualities, disrupt vibration and phase closure, and eventually lead to phase asymmetry ${ }^{33}$ resulting in increased jitter and shimmer. Furthermore, acute inflammation may prevent the vocal folds from closing properly, resulting in air leakage and decreased HNR..$^{30,33}$ In some individuals, videostroboscopic studies have revealed an incomplete glottal closure, ${ }^{19}$ anterior and posterior chinks, ${ }^{19,25}$ or hourglass ${ }^{25}$ and spindle-shaped vibratory closure pattern ${ }^{21,25}$ following a loading task. The increased perturbation parameters (jitter and shimmer) may also be explained by muscle fatigue, resulting in decreased stability of vocal fold posturing and tension during sustained phonation. For all these reasons, this third group is hypothesized to present acoustic and functional perturbations, which may result from acute inflammation and muscle fatigue following vocal loading through the workday.

Because one individual may belong to different groups depending on the time of year (intra-speaker variability), demographic data were not taken into account for statistical analyses. However, visual examination of Figure 2 shows that six teachers remained in the same group at all three times of year. Four of them always belonged to group 1 (S6, S7, S9, and S12); S7 and S9 did not report any particular complaints in the questionnaire, but S6 and S12 had previously experienced voice problems during their career. Two subjects always belonged to group 3: S10, who reported difficulty reaching high notes while singing, and S18, who did not report any particular complaints in the questionnaire.

\section{LIMITATIONS, PERSPECTIVES, AND CONCLUSIONS}

The physiological interpretations of the observed acoustic patterns presented above remain hypotheses. They require additional investigations to be confirmed. The hypothesis of vocal hyperfunction for the second type of voice change pattern during the workday requires physiological measures of laryngeal tension, using electromyography ${ }^{18}$ or vocal fold kinematics. ${ }^{34}$ Similarly, laryngeal examination is necessary to confirm the hypothesis that acute inflammation or vocal fold closure defaults followed vocal loading in the third group. However, these measures may be difficult to obtain in real-life situations.

In future studies attempting to identify different behaviors, other sensitive descriptors of vocal load should be considered, such as phonation threshold pressure and inability to produce soft voice. ${ }^{1,11,16}$ Self-assessments of vocal load symptoms may also be used, including the perceived phonatory effort ${ }^{1,11}$ and specific questionnaires (eg, the Vocal Fatigue Index ${ }^{35}$ ). Because not all voice changes during a teaching day can be interpreted as the consequence of vocal load, future research should investigate the exact relation between the amount of voice and the voice changes during the workday, by means of dosimetry. Finally, measuring voice changes in a control group that does not use the voice as a professional tool may help to differentiate loading from potential co-factors such as natural voice changes during the day.

To conclude, the present study shows that teachers demonstrate varying voice patterns during a workday. Based on acoustic measures, the typology presented was a first step in targeting 
individuals engaging in potentially harmful vocal behaviors, namely groups 2 and 3 . A second step would be to identify the intrinsic and extrinsic factors contributing to an individual's voice changes during a workday, in an attempt to offer tailored strategies to prevent the individual from developing voice disorders.

Different methods have proven efficacious in the treatment of vocal load symptoms, such as chant therapy, ${ }^{23}$ resonant voice therapy ${ }^{36}$ low-level light therapy ${ }^{16}$ and vocal rest. ${ }^{36}$ Accordingly, a final step would be to develop efficient prevention and treatment methods according to the type of voice changes during the workday.

\section{Acknowledgments}

We thank the Mutuelle Générale de l'Education Nationale (MGEN) and Grenoble's education authority for their help in recruiting the participants. A.R. was supported by a Marie Curie COFUND postdoctoral grant of the University of Liège (Belgium) and the European Union. This funding source was not involved in the collection, analysis, and interpretation of data; in the writing of the present article; or in the decision to submit the article for publication.

\section{REFERENCES}

1. Fujiki RB, Sivasankar MP. A review of vocal loading tasks in the voice literature. J Voice. 2017;31:388.e33-388.e39. doi:10.1016/j.jvoice.2016 .09 .019 .

2. Remacle A, Finck C, Roche A, et al. Vocal impact of a prolonged reading task at two intensity levels: objective measurements and subjective selfratings. $J$ Voice. 2012;26:177-186. doi:10.1016/j.jvoice.2011.07.016.

3. Remacle A, Morsomme D, Finck C. Comparison of vocal loading parameters in kindergarten and elementary school teachers. J Speech Lang Hear Res. 2014;57:406-415.

4. Kristiansen J, Lund SP, Persson R, et al. A study of classroom acoustics and school teachers' noise exposure, voice load and speaking time during teaching, and the effects on vocal and mental fatigue development. Int Arch Occup Environ Health. 2014;1-10.

5. Rantala L, Vilkman E, Bloigu R. Voice changes during work: subjective complaints and objective measurements for female primary and secondary schoolteachers. J Voice. 2002;16:344-355.

6. Laukkanen A-M, Ilomaki I, Leppanen K, et al. Acoustic measures and self-reports of vocal fatigue by female teachers. J Voice. 2008;22:283-289.

7. Solomon NP. Vocal fatigue and its relation to vocal hyperfunction. Int J Speech Lang Pathol. 2008;10:254-266. doi:10.1080/14417040701730990.

8. Vilkman E. Occupational safety and health aspects of voice and speech professions. Folia Phoniatr Logop. 2004;56:220-253.

9. Titze IR. Vocal fatigue: Some biomechanical considerations. In: Lawrence VL, ed. Transcripts of the Twelfth Symposium: Care of the Professional Voice. New York: The Julliard School; 1984:97-104.

10. Titze IR. Principles of Voice Production. 2nd ed. Iowa City, IA: National Center for Voice and Speech; 2000.

11. Chang A, Karnell MP. Perceived phonatory effort and phonation threshold pressure across a prolonged voice loading task: a study of vocal fatigue. J Voice. 2004;18:454-466.

12. Enflo L, Sundberg J, McAllister A. Collision and phonation threshold pressures before and after loud, prolonged vocalization in trained and untrained voices. $J$ Voice. 2013;27:527-530. doi:10.1016/j.jvoice.2013 .03 .008 .

13. Welham NV, Maclagan MA. Vocal fatigue: current knowledge and future directions. J Voice. 2003;17:21-30.
14. Nanjundeswaran C, VanSwearingen J, Verdolini Abbott K. Metabolic mechanisms of vocal fatigue. J Voice. 2017;31:378.e1-378.e11. doi:10.1016/ j.jvoice.2016.09.014.

15. Sivasankar M, Erickson E, Schneider S, et al. Phonatory effects of airway dehydration: preliminary evidence for impaired compensation to oral breathing in individuals with a history of vocal fatigue. J Speech Lang Hear Res. 2008;51:1494-1506. doi:10.1044/1092-4388(2008/07-0181).

16. Kagan LS, Heaton JT. The effectiveness of low-level light therapy in attenuating vocal fatigue. J Voice. 2017;31:384.e15-384.e23. doi:10.1016/ j.jvoice.2016.09.004.

17. Remacle A, Morsomme D, Berrué E, et al. Vocal impact of a prolonged reading task in dysphonic versus normophonic female teachers. $J$ Voice. 2012;26:820. doi:10.1016/j.jvoice.2012.06.002.

18. Boucher VJ, Ayad T. Physiological attributes of vocal fatigue and their acoustic effects: a synthesis of findings for a criterion-based prevention of acquired voice disorders. J Voice. 2010;24:324-336.

19. Stemple JC, Stanley J, Lee L. Objective measures of voice production in normal subjects following prolonged voice use. J Voice. 1995;9:127-133.

20. Remacle A, Schoentgen J, Finck C, et al. Impact of vocal load on breathiness: perceptual evaluation. Logoped Phoniatr Vocol. 2014;39:139-146.

21. Solomon NP, DiMattia MS. Effects of a vocally fatiguing task and systemic hydration on phonation threshold pressure. J Voice. 2000;14:341-362.

22. Murray ES, Hands GL, Calabrese CR, et al. Effects of adventitious acute vocal trauma: relative fundamental frequency and listener perception. $J$ Voice. 2016;30:177-185. doi:10.1016/j.jvoice.2015.04.005.

23. McCabe DJ, Titze IR. Chant therapy for treating vocal fatigue among public school teachers: a preliminary study. Am J Speech Lang Pathol. 2002;11:356-369.

24. Dejonckere $\mathrm{PH}$, Bradley $\mathrm{P}$, Clemente $\mathrm{P}$, et al. A basic protocol for functional assessment of voice pathology, especially for investigating the efficacy of (phonosurgical) treatments and evaluating new assessment techniques. Eur Arch Otorhinolaryngol. 2001;258:77-82.

25. Buekers R. Are voice endurance tests able to assess vocal fatigue? Clin Otolaryngol. 1998;23:533-538.

26. Boersma P, Weenink D. Praat: Doing Phonetics by Computer; 2017. http://www.praat.org. Accessed January 23, 2017.

27. Titze IR. On the relation between subglottal pressure and fundamental frequency in phonation. J Acoust Soc Am. 1989;85:901-906.

28. Jonsdottir V, Laukkanen A-M, Vilkman E. Changes in teachers' speech during a working day with and without electric sound amplification. Folia Phoniatr Logop. 2002;54:282-287.

29. Baken RJ, Orlikoff RF. Clinical Measurement of Speech and Voice. Clifton Park, NY: Cengage Learning; 2000.

30. Kreiman J, Sidtis D. Foundations of Voice Studies: An Interdisciplinary Approach to Voice Production and Perception. Boston: John Wiley \& Sons; 2011.

31. Roy N, Fetrow RA, Merrill RM, et al. Exploring the clinical utility of relative fundamental frequency as an objective measure of vocal hyperfunction. J Speech Lang Hear Res. 2016;59:1002-1017. doi:10.1044/2016_jslhr-s$15-0354$.

32. Scherer R, Titze I, Raphael B, et al. Vocal fatigue in a trained and an untrained voice user. In: Baer T, Sasaki C, Harris K, eds. Laryngeal Function in Phonation and Respiration. San Diego, CA: Singular Publishing.; 1987:533-555.

33. Altman KW. Vocal fold masses. Otolaryngol Clin North Am. 2007;40:10911108.

34. Stepp CE, Hillman RE, Heaton JT. A virtual trajectory model predicts differences in vocal fold kinematics in individuals with vocal hyperfunction. J Acoust Soc Am. 2010;127:3166-3176. doi:10.1121/1.3365257.

35. Nanjundeswaran C, Jacobson BH, Gartner-Schmidt J, et al. Vocal fatigue index (VFI): development and validation. J Voice. 2015;29:433-440. doi:10.1016/j.jvoice.2014.09.012.

36. Verdolini Abbott K, Li NYK, Branski RC, et al. Vocal exercise may attenuate acute vocal fold inflammation. J Voice. 2012;26:814.e1-814.e13. doi:10.1016/j.jvoice.2012.03.008. 